\title{
MENINGEAL SYNDROME IN THE PRACTICE OF INFECTIOUS DISEASES SPECIALISTS
}

\author{
Aleksandar Ranković ${ }^{1}$, Miodrag Vrbić, ${ }^{1,2}$, Maja Jovanović, ${ }^{1,}$, Lidija \\ Popović-Dragonjić ${ }^{1,2}$, Marina Đorđević-Spasić ${ }^{1}$
}

\author{
Clinic for Infectious Diseases, Clinical Centre Niš, Niš, Serbia \\ University of Niš, Faculty of Medicine, Niš, Serbia ${ }^{2}$ \\ Contact: Aleksandar Ranković \\ Branka Krsmanovića 5/17, 18000 Niš, Serbia \\ E-mail: drarankovic@gmail.com
}

Central nervous system (CNS) infections are among the most dramatic medical conditions, not just because of their clinical presentations, possible ultimate consequences, but also because of the complexity of their diagnosis and treatment, and clinically they are manifested with meningeal syndrome, regardless of the type of causative agent. The aim of the study was to determine the correlation between certain clinical signs of meningeal syndrome and results of lumbar puncture which is used to diagnose the central nervous system infection. The study included a group of 54 patients who were treated at the Clinic for Infectious Diseases Clinical Centre Niš, with a clinical picture of bacterial meningitis.

The diagnosis of the disease was based on a clinical picture, findings of lumbar puncture and isolation of the causative agent out of the cerebrospinal fluid. In all patients, there was pleocytosis in the cerebrospinal fluid with predomination of polymorphonuclears. There were $20(37.0 \%)$ females and $34(63.0 \%)$ males, with mean age $52.37 \pm 18.10$ years. The most dominant clinical symptoms in patients were headache in $74.1 \%$, elevated temperature in $70.4 \%$, stiff neck in $63.0 \%$ and Brudzinski's upper sign in $55.6 \%$.

Ethical verification from the cerebrospinal fluid was negative in $32(59.3 \%)$ patients, Klebsiella, Pneumoccocus and Staphylococcus were found in $4(7.4 \%)$ patients each. It was found that headaches with stiff neck, disturbance of consciousness and elevated temperature represent an absolute indication for lumbar puncture and are in direct correlation with the positive finding in the cerebrospinal fluid. Acta Medica Medianae 2017;56(2):32-37.

Key words: CNS infection, meningitis, meningeal syndrome, lumbar puncture 